\title{
Growth and characterization of 7,7,8,8-tetracyano-quinodimethane crystals on chemical vapor deposition graphene
}

\author{
Andrés Black ${ }^{1,2, b a}$, Fernando Jiménez ${ }^{1}$, Ramón Bernardo-Gavito ${ }^{1, b}$, Santiago \\ Casado $^{1}$, Daniel Granados ${ }^{1}$, Amadeo L. Vázquez de Parga ${ }^{1,2}$ \\ ${ }^{1}$ IMDEA Nanociencia, Calle Faraday 9, Cantoblanco 28049, Madrid, Spain \\ ${ }^{2}$ Departamento de Física de la Materia Condensada, Universidad Autónoma de Madrid, Cantoblanco \\ 28049, Madrid, Spain
}

Chemical functionalization of graphene could pave the way for favorably modifying its already remarkable properties. Organic molecules have been utilized to this end as a way to alter graphene's structural, chemical, electrical, optical and even magnetic properties. One such promising organic molecule is 7,7,8,8-tetracyano-quinodimethane (TCNQ), a strong electron acceptor which has been shown to be an effective $p$-dopant of graphene. This study explores the thermal evaporation of TCNQ onto graphene transferred onto $\mathrm{SiO}_{2} / \mathrm{Si}$ substrates. Using two different home-made thermal evaporators, a wide range of TCNQ growth regimes are explored, from thin films to bulk crystals. The resulting graphene/TCNQ structure is characterized via optical microscopy, Raman spectroscopy and atomic force microscopy (AFM). Films are found to be comprised of TCNQ and the oxidized product of TCNQ, $\alpha, \alpha$-dicyano-p-toluoylcyanide (DCTC), which confirms the electron charge transfer from graphene to the TCNQ films. AFM measurements of these films show that after forming a rather smooth layer covering the graphene surface, small clusters start to form. For higher TCNQ coverage, the clusters agglomerate, becoming quite large in size and forming ripples or wrinkles across the surface.

Keywords: A1. Diffusion, A1. Nanostructures, A3. Physical vapor deposition processes, B1. Organic compounds

\section{INTRODUCTION}

In the past decade extensive work has been carried out towards the goal of chemically functionalizing graphene to enhance and complement its remarkable properties ${ }^{1}$. Organic molecules have been used to chemically functionalize through both covalent ${ }^{2}$ and non-covalent means $^{3}$. TCNQ is an organic molecule that has been successfully used to non-covalently functionalize graphene. A strong electron acceptor, TCNQ has been utilized to produce highly $p$-doped graphene, resulting in the opening of an electrical bandgap in bilayer graphene ${ }^{4}$, and for use as a conductive anode in graphene organic solar cells ${ }^{5}$. Recent studies carried out in ultra-high vacuum have found that TCNQ deposited onto graphene grown epitaxially on ruthenium is able to form extended spin-split electronic bands resulting in long range magnetic order $^{3}$. X-ray photoemission spectroscopy (XPS) and scanning tunneling microscopy (STM) measurements confirmed that charge transfer from the ruthenium substrate to the TCNQ, modulated by the graphene layer in between, caused the molecules to develop a magnetic moment ${ }^{6,7}$.

TCNQ and its salts have been studied extensively by Raman spectroscopy, confirming their strong electron accepting capabilities for both films and crystals synthesized by evaporation or

\footnotetext{
${ }^{a}$ Author to whom correspondence should be addressed: andres.black@uam.es

${ }^{\mathrm{b}}$ Currently Physics Department, Lancaster University, LA1 4YB, Lancaster, United Kingdom 
from solution ${ }^{8-13}$. Depending on the growth conditions and substrate, the oxidation product of TCNQ $^{2-}, \alpha, \alpha$-dicyano-p-toluoylcyanide (DCTC) is often detected. For TCNQ films evaporated onto Ag, DCTC was detected for thin films, suggesting that the layers close to the Ag substrate were ionized and oxidized. As the TCNQ film thickness increased, DCTC was no longer detected, suggesting that the upper layers of the film were comprised of neutral TCNQ ${ }^{14}$. Similar behavior has been observed via XPS measurements for $\mathrm{F}_{4}$-TCNQ molecules deposited on epitaxial graphene on SiC. Electron transfer from the graphene to the first few layers of adsorbed $\mathrm{F}_{4}$-TCNQ was found to $p$-dope the graphene ${ }^{15}$. For CVD grown graphene transferred to an insulating substrate, DCTC was also detected for low coverage, whereas only TCNQ was detected for higher coverages, suggesting a similar mechanism of electron charge transfer from the graphene substrate to the first few TCNQ layers adjacent to it ${ }^{16}$.

This study seeks to understand the growth process and morphology of TCNQ evaporated onto Chemical Vapor Deposition (CVD) grown graphene transferred onto $\mathrm{SiO}_{2} / \mathrm{Si}$ substrate, which is commonly used for device fabrication. Understanding the growth mechanism of TCNQ on graphene is necessary for the further development of devices based on graphene/TCNQ structures, with the hope of exploiting the unique properties of each material. TCNQ films have been shown to be effective graphene dopants as well as exhibiting magnetic properties ${ }^{3}$, whereas TCNQ crystals have been shown to have unique electrical ${ }^{17}$ and optoelectronic ${ }^{18}$ properties when interfaced with adequate complementary organic crystals. Different growth regimes of TCNQ on graphene were explored by varying the sublimation temperature, substrate temperature and vapor concentration of the TCNQ. This resulted in various TCNQ/graphene structures, ranging from thin films to pure TCNQ crystals. Electron charge transfer from the graphene to the TCNQ and subsequent oxidation to DCTC, as previously seen by Qi et $\mathrm{al}^{16}$, was confirmed for thin films via Raman spectroscopy. Atomic force microscopy measurements (AFM) showed that at low coverage, the TCNQ films seems to have almost no effect on the graphene surface, whereas for increasing coverage, clusters form, growing in size to the point that they strain the graphene and form filament-like wrinkles.

\section{METHODS}

Graphene was grown on $25 \mu \mathrm{m}$ thick polycrystalline copper foil via chemical vapor deposition in a CVD furnace. The foil was heated to $1000^{\circ} \mathrm{C}$ while flowing 100 standard cubic centimeters $(\mathrm{sccm})$ hydrogen. The temperature was allowed to stabilize for 10 minutes, before increasing the hydrogen flow to $500 \mathrm{sccm}$ and introducing $1 \mathrm{sccm}$ methane for 25 minutes in order to induce graphene growth. The furnace was then rapidly cooled to room temperature while flowing $100 \mathrm{sccm}$ hydrogen. The entire process was carried out at $10 \mathrm{mbar}$. Graphene was then

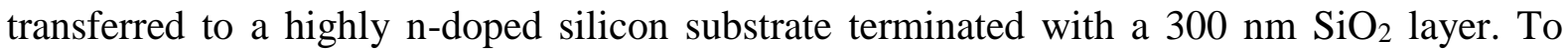
achieve the transfer, a PMMA layer was spun on the copper foil (about $180 \mathrm{~nm}$ thickness), before etching the copper foil in an ammonium persulfate etchant $(0.1 \mathrm{~g} / \mathrm{mL}$ in deionized water). After rinsing the PMMA/graphene stack several times in deionized water, the stack was scooped out with the target $\mathrm{Si} / \mathrm{SiO}_{2}$ wafer. The graphene was allowed to dry over night before removing the PMMA layer by rinsing in acetone. Thermal annealing was then carried out at $300^{\circ} \mathrm{C}$ in $\mathrm{Ar} / \mathrm{H}_{2}$ 96/4\% in order to remove remaining PMMA residues. Raman spectroscopy measurements were carried out using a $488 \mathrm{~nm}$ laser, with a spot size of about $1 \mu \mathrm{m}$ using an x80 objective and collected in a cooled silicon CCD detector (Andor Newton), with a $0.5 \mathrm{~m}$ spectrometer length and a $300 \mathrm{l} / \mathrm{mm}$ grating. 


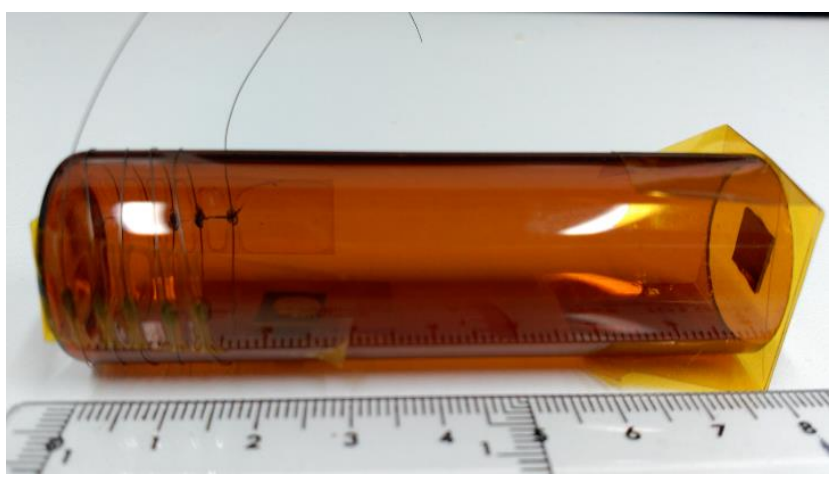

Figure 1 Picture of large tube TCNQ evaporator with aluminum foil removed from exterior. Samples are placed at the tube mouth and inside the tube, $2 \mathrm{~cm}$ away from tube bottom. In the small tube evaporator, the sample is placed only on the mouth of the tube.

TCNQ evaporation was carried out in a quartz tube placed inside a vacuum desiccator, at a pressure of 600 mbar. For each growth one of two quartz tubes were used, a small one $(3.5 \mathrm{~cm}$ long, $1.8 \mathrm{~cm}$ diameter) or a large one $(8 \mathrm{~cm}$ long, $2.3 \mathrm{~cm}$ diameter $)$, shown in Figure 1. The lower region of both quartz tubes were heated resistively, by coiling tungsten wire around the exterior of the tubes. $100 \mathrm{mg}$ TCNQ powder (Sigma Aldrich 157635) was placed inside the bottom of the tube. In order to achieve more homogeneous heating, aluminum foil was also wrapped around the bottom $2 \mathrm{~cm}$ of the tubes. An in situ thermocouple was used to calibrate the relation between the power dissipated in the resistive coil (usually between 2 and $10 \mathrm{~W}$ ) and the temperature at the bottom of the tube, and subsequent evaporations were carried out without the thermocouple. The quartz tubes were sealed at their mouth openings with a glass slide, with the graphene/ $\mathrm{SiO}_{2}$ samples mounted on the glass slide facing the TCNQ powder inside the tube. In the case of the large evaporator, apart from at the tube mouth, samples were also mounted inside the quartz tube $2 \mathrm{~cm}$ away from the tube bottom, facing the TCNQ powder, as shown in Figure 1.

\section{RESULTS AND DISCUSSION}

Figure 2 shows white light reflection optical images of TCNQ evaporated onto graphene/SiO $\mathrm{S}_{2}$ for different sublimation temperatures and growth times in the small tube evaporator. As seen in Figure 2(a), a growth temperature of $130^{\circ} \mathrm{C}$ for 75 minutes results in the formation of pelletlike crystals on the surface of the graphene, with lateral dimensions on the order of $5 \mu \mathrm{m}$. These crystals are separated by a typical distance of a few dozen microns, indicating the high mobility of the individual TCNQ molecules on graphene. Increasing the growth temperature to $160^{\circ} \mathrm{C}$ and maintaining the same growth time clearly changes the growth regime, as shown in Figure 2(b). The graphene substrate is no longer visible and seems to have been covered by a thick film. The morphology of the crystals on the surface has also changed, from pellets to needles with a typical length on the order of $10 \mu \mathrm{m}$. The needles are packed more closely together on the surface than the pellets, with many needles touching each other. It seems that the rise in the sublimation temperature substantially modifies the density of TCNQ molecules on the surface. Now the molecules are no longer able to reach the initial condensation points, on the contrary the TCNQ molecules self-assemble, probably via $\mathrm{H}$-bonds ${ }^{3}$, forming a full layer that reduces the effective diffusion length of the incoming molecules and as consequence increases the density of 3D islands. From this temperature the growth mode remains unchanged and further increasing the growth temperature to $180^{\circ} \mathrm{C}$ completely saturates the graphene surface with 
TCNQ needles, as shown in Figure 2(c), despite a reduction in the evaporation time from 75 to 45 minutes. Evaporating at the highest temperature obtainable in the small tube evaporator, $200^{\circ} \mathrm{C}$, for 45 minutes, maintains the high crystal density on the surface but results in a change in the structure of the crystals, as seen in Figure 2(d). Apart from needles, many plaque-shaped crystals are seen on the surface, with a typical size of 5 to $10 \mu \mathrm{m}$.
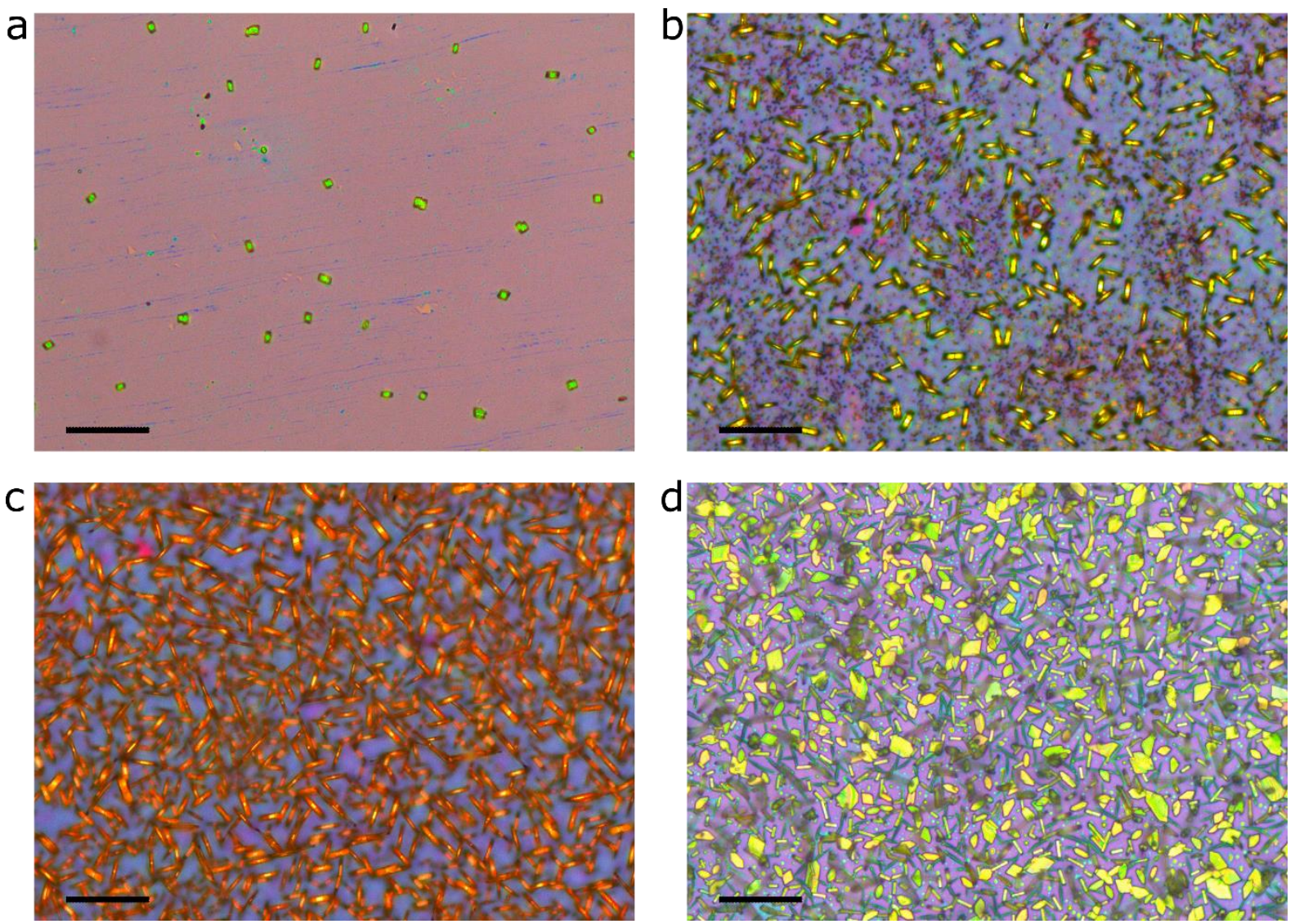

Figure 2 Optical images of TCNQ evaporated onto graphene/SiO $/ 2$ Si substrate in small tube evaporator with the following conditions: (a) 75 minutes at $130^{\circ} \mathrm{C}$, (b) 75 minutes at $160^{\circ} \mathrm{C}$, (c) 45 minutes at $180^{\circ} \mathrm{C}$ and (d) 45 minutes at $200^{\circ}$. All scale bars $25 \mu \mathrm{m}$.

The large tube evaporator (diameter $2.3 \mathrm{~cm}$ and length $8 \mathrm{~cm}$ ) can accommodate two samples simultaneously, one at the extreme of the tube, where the sample remains close to room temperature, and another one inside the tube close to the resistive heating coils, where the sample is heated along with the TCNQ powder. This configuration allows for the comparison of the TCNQ film growth at different substrate temperatures for the same evaporation run. Figure 3 shows optical images of TCNQ evaporated onto graphene/SiO $\mathrm{S}_{2}$ for different growth conditions in the large tube evaporator. Figure 3(a), (b) show the images corresponding to the substrate placed at the mouth of the tube, where it remains close to room temperature. Evaporating at $120^{\circ} \mathrm{C}$ for 60 minutes results in the formation of TCNQ crystal pellets, as seen in Figure 3(a). Figure 3(b) shows that increasing the growth temperature to $170^{\circ} \mathrm{C}$ favors the formation of short needles on the graphene surface, with a typical length of 2 to $3 \mu \mathrm{m}$, in a very dense configuration, with most needles touching another needle. The comparison with images shown in Figure 2(a), (b) reveals a similar morphology for both growth conditions. When the substrate remains close to room temperature, the main findings can summarized as follows: for lower sublimation temperatures the growth of pellet-like TCNQ crystals occurs, whereas for 
higher sublimation temperatures, the growth regime changes, with the TCNQ molecules on the surface forming needles that are closer to each other than the pellets.
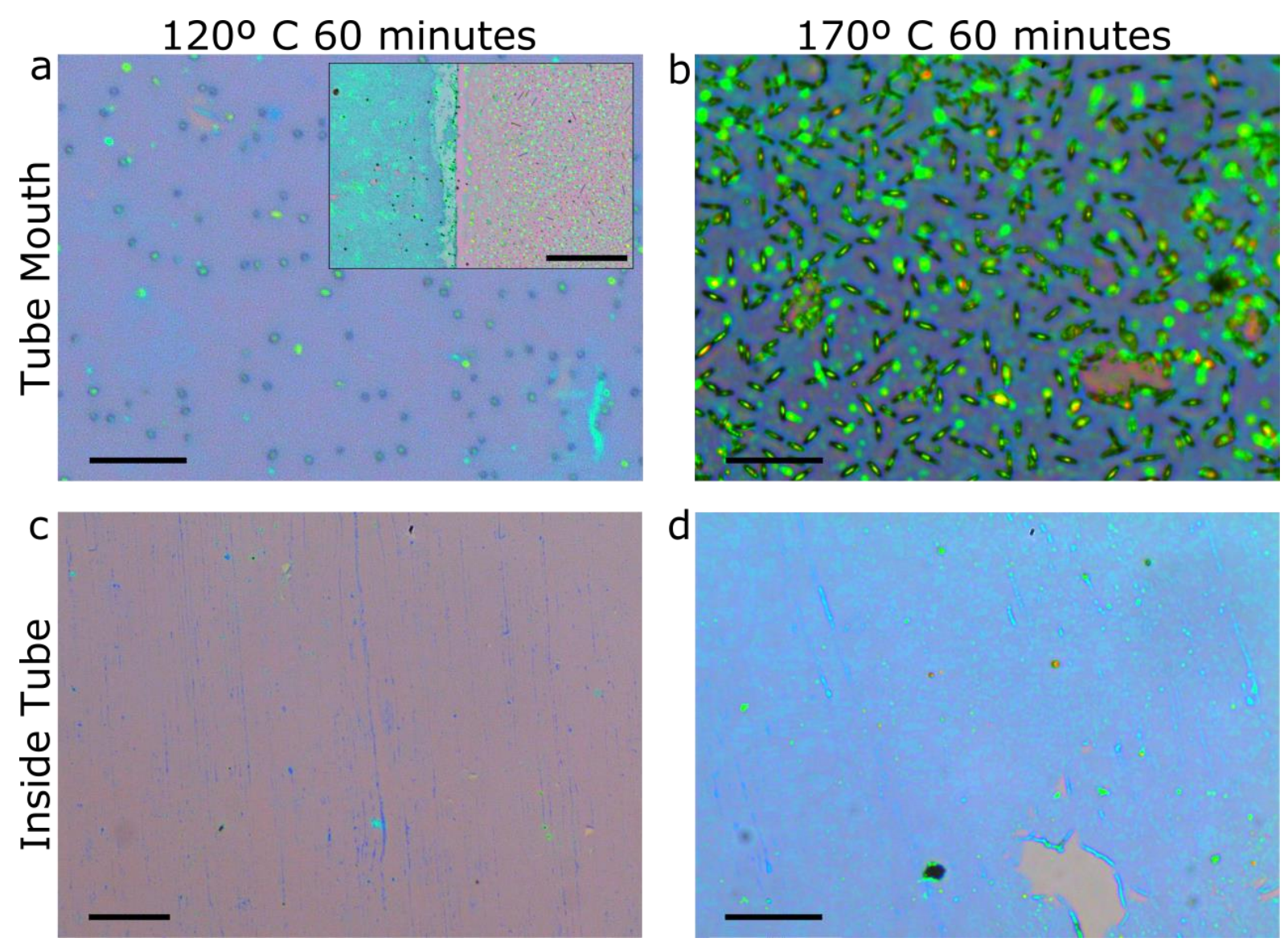

Figure 3 Optical images of TCNQ evaporated onto graphene/ $\mathrm{SiO}_{2} / \mathrm{Si}$ substrate in large tube evaporator with the conditions outlined on the margins of the images. Scale bar in (c) is $40 \mu \mathrm{m}$, (a), (b) and (d) are $15 \mu \mathrm{m}$. Inset: Same sample as in a, showing the boundary between graphene (left) and bare $\mathrm{SiO}_{2}$ (right). TCNQ crystals can be seen on both sides of the boundary. Scale bar $250 \mu \mathrm{m}$.

It is worth noting that the samples obtained for the evaporation done in the small tube and in the large tube with the substrate place at the mouth present differences in the crystal coverages obtained, despite similar growth conditions and substrate temperatures. The main difference is the higher crystal coverage obtained with the small tube compared to substrates on the mouth of the large tube. The reason for this behavior is not completely clear, since the vapor pressure and hence concentration of TCNQ molecules at the same evaporation temperature should be equal regardless of the tube size. One possible reason for the different crystal coverage is that the TCNQ molecules have more surface area upon which they can be deposited in the large tube. If a larger proportion of the TCNQ molecules are sticking to the tube walls in the large tube, then fewer TCNQ molecules will end up reaching the substrate at the mouth of the tube, despite the fact that the theoretical TCNQ vapor pressure is the same at a given temperature. In addition, the substrate in the small tube is fairly close to the heat source and may be heated slightly above ambient, whereas the substrate at the mouth of the large tube is far from the heat source and therefore remains at ambient temperature. This difference in substrate temperature could be an additional factor in explaining the differences in growth regime. 
For samples placed on the inside of the large tube, where the substrate is heated during the evaporation, no TCNQ crystals were formed for any of the growth conditions, only TCNQ films. For low TCNQ coverage, like that shown in Figure 3(c), practically no changes are noticeable on the graphene surface when compared to pristine graphene. By increasing the evaporation temperature from $120^{\circ} \mathrm{C}$ to $170^{\circ} \mathrm{C}$, a change is observed in the coloration of the graphene, which becomes slightly bluer as seen in Figure 3(d) (note that in all of these images, the white balance has been adjusted to make bare $\mathrm{SiO}_{2}$ background equal). More interestingly, blue patches and spots are visible amongst the purple background, covering almost half of the surface area and with a typical size of 15 to $20 \mu \mathrm{m}$.

From these results it is clear that the growth mechanism on both the large and small tube evaporator is the same for the substrates held close to room temperature, and leads to the formation of TCNQ crystals. When the substrate is held at elevated temperatures during evaporation the TCNQ deposition produces a uniform thin molecular film on the graphene substrate. This change in the structure of the resulting sample may be due to two different mechanisms. Firstly, an elevated substrate temperature increases desorption, reducing the overall sticking coefficient of the TCNQ molecules present on the graphene surface. Secondly, TCNQ molecules on the elevated temperature substrate will have more thermal energy to diffuse about the surface, making it less likely they will remain stuck to previously formed TCNQ clusters they encounter, thereby limiting the formation of large crystals. Desorption of the TCNQ molecules from the substrates held at high temperature during the growth can be checked by evaporating TCNQ molecules on bare $\mathrm{SiO}_{2}$ surface. On this surface, a shorter diffusion length is expected in comparison to graphene. For growths with the substrate at high temperatures, no TCNQ is detected on the $\mathrm{SiO}_{2}$ surface, either via optical inspection or Raman spectroscopy, indicating that the sticking coefficient for TCNQ on $\mathrm{SiO}_{2}$ at elevated temperatures drops to zero. At low substrate temperatures, however, TCNQ crystals form on the $\mathrm{SiO}_{2}$ surface in much the same way as they do on the graphene surface, as can be seen by the inset in Figure 3(a). For both graphene and SiO2 surfaces, the TCNQ sticking coefficient is high for low substrate temperatures and low for high substrate temperatures.

Raman spectroscopy measurements were carried out of the TCNQ/graphene structures, with spectra from growths in the small tube shown in Figure 4(a), and spectra from the films grown on the inside of the large tube shown in Figure 4(b). A reference spectrum of pristine graphene on $\mathrm{SiO}_{2}$ is also shown in Figure 4(a), exhibiting the typical 2D peak near $2700 \mathrm{~cm}^{-1}$ and $\mathrm{G}$ peak near $1585 \mathrm{~cm}^{-1}$. The very low intensity of the D peak, located near $1350 \mathrm{~cm}^{-1}$ and associated with disorder in the graphene lattice, confirms the low density of defects in graphene, while the large ratio between the intensities of the $2 \mathrm{D}$ and $\mathrm{G}$ peaks confirms that the graphene is monolayer ${ }^{19}$. The red curve in Figure 4(a) was taken between the crystals on the surface of the sample from Figure 2(a). This spectrum continues to show the graphene 2D and G peaks, but with a diminished intensity in the ratio between the two peaks compared to the pristine graphene spectrum. The peaks observed at 1179, 1288 and $1621 \mathrm{~cm}^{-1}$ are associated with the presence of DCTC, the oxidation product of TCNQ, replicating the measurements made in Qi et $\mathrm{al}^{16}$. In addition to the DCTC, the presence of TCNQ on the graphene surface is confirmed from the peaks at 1204,1446 , and $2218 \mathrm{~cm}^{-18,12,9}$. The peak located at $1592 \mathrm{~cm}^{-1}$ results from a combination of the graphene $\mathrm{G}$ peak and a TCNQ peak at $1598 \mathrm{~cm}^{-1}$. An increase in the graphene D peak is also observed, suggesting that the graphene is more disordered after the TCNQ evaporation. The presence of both TCNQ and DCTC on the surface in between crystal pellets confirms that electron charge transfer between the graphene and the TCNQ molecules is occurring. The blue curve in Figure 4(a) also shows Raman spectra taken on top of the 
crystals from Figure 2(a) (blue curve). Strong, pure TCNQ peaks are detected, with no sign of graphene or DCTC, with identical results obtained whenever Raman spectra were taken on top of needle or pellet-like crystals.
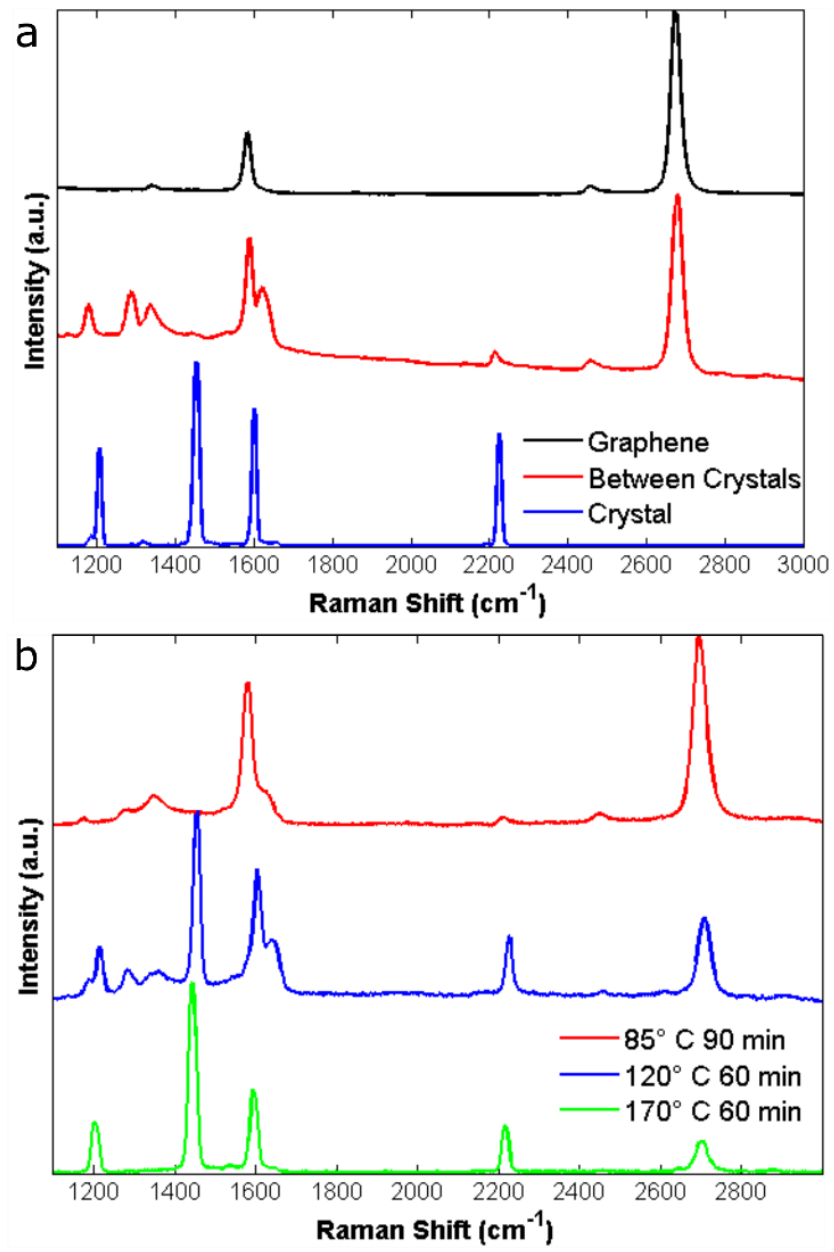

Figure 4 Raman spectra of pristine graphene on $\mathrm{SiO}_{2}$, as well as TCNQ evaporated onto graphene with different conditions in (a) mouth of small tube evaporator and (b) inside of large tube evaporator.

The information gathered from the optical image in Figure 2(a) and the corresponding Raman spectra between and on top of the crystals would suggest that as a TCNQ/DCTC film forms on the graphene surface, microscopic clusters of TCNQ crystals are created and grow to become ever larger TCNQ crystals. It is possible that defects, rips, tears or foreign impurities on the graphene surface act as heterogeneous nucleation sites for the formation of the TCNQ clusters. These TCNQ clusters grow into crystals by incorporating TCNQ molecules both directly from the atmosphere and from diffusing TCNQ molecules on the substrate surface, which become absorbed into the TCNQ crystals when they come into contact with it. As evaporation temperatures are increased, an increase in the nucleation density of the TCNQ crystals is observed, as well as a saturation of the graphene surface with a thick TCNQ film. Additionally, instead of forming pellets, the formation of needle-like TCNQ crystals is favored, as has been observed in the vapor growth of other organic crystals ${ }^{20,21}$.

Raman spectra taken of samples grown on the inside of the large tube, where the substrate is heated during growth, shown in Figure $4 \mathrm{~b}$, detect the presence of TCNQ and DCTC covering the entire surface for evaporation temperatures as low as $85^{\circ} \mathrm{C}$. At this low temperature (red 
curve in Figure 4(b)), the graphene peaks are quite strong, and weak peaks corresponding mostly to DCTC are identifiable in the range between 1150 and $1300 \mathrm{~cm}^{-1}$, as well as a TCNQ peak at $2220 \mathrm{~cm}^{-1}$. A slight increase in the graphene D peak is also observed. Increasing the evaporation temperature to $120^{\circ} \mathrm{C}$, corresponding to the sample shown in Figure 3(c), intensifies both TCNQ and DCTC peaks with respect to the graphene peaks, although an increase in the $\mathrm{D}$ peak is also appreciable. For the highest achievable evaporation temperature in the large tube evaporator, $170^{\circ} \mathrm{C}$, corresponding to the sample shown in Figure 3(d), only TCNQ peaks are detectable, along with a weakened graphene 2D peak, with no sign of DCTC. It would seem that for these high coverages, the bulk TCNQ film is thick enough that it overwhelms any signal that may be coming from the oxidized layers of DCTC adjacent to the graphene, where charge transfer is occurring. The measured $\mathrm{G}$ peak frequency is seen to increase from around $1585 \mathrm{~cm}^{-1}$ in pristine graphene towards $1600 \mathrm{~cm}^{-1}$ for samples grown in both the large and small evaporator. This is another indication that there is charge transfer from the graphene to the TCNQ molecules, resulting in graphene p-doping ${ }^{22,23}$. Apart from the charge transfer, part of this apparent shift in the graphene $\mathrm{G}$ peak may also be due to its merging with the TCNQ peak situated at $1600 \mathrm{~cm}^{-1}$.

In order to clarify the structure of the graphene/TCNQ films, and what might be causing the slight increase in the graphene D peak upon TCNQ evaporation, AFM measurements were carried out of the TCNQ films grown with the substrate at elevated temperatures. The resulting images are shown in Figure 5. A pristine graphene surface, after thermal annealing to remove PMMA residues, is shown in Figure 5(a). Some folds are visible along the graphene, with a typical height between 2 and $3 \mathrm{~nm}$. A few much larger PMMA clusters remain on the film, with heights varying between 20 and $30 \mathrm{~nm}$, although the surface is mostly clean. The RMS value of the region outlined by the blue 1.5 by $1.5 \mu \mathrm{m}$ square shown in the figure is $0.73 \mathrm{~nm}$. For evaporation at low temperatures (between $85^{\circ} \mathrm{C}$ and $110^{\circ} \mathrm{C}$ ) up to 120 minutes, practically no change was discernible in the surface when compared to pristine graphene. Increasing the evaporation temperature to $120^{\circ} \mathrm{C}$ produces an obvious change on the graphene surface, as seen in Figure 5(b), with the appearance of small TCNQ/DCTC clusters. These clusters seem to be randomly dispersed about the graphene surface, aggregating in groups with a typical size of 50 to $200 \mathrm{~nm}$, and heights between 2 and $3 \mathrm{~nm}$. The RMS value of the blue square is $1.1 \mathrm{~nm}$. 

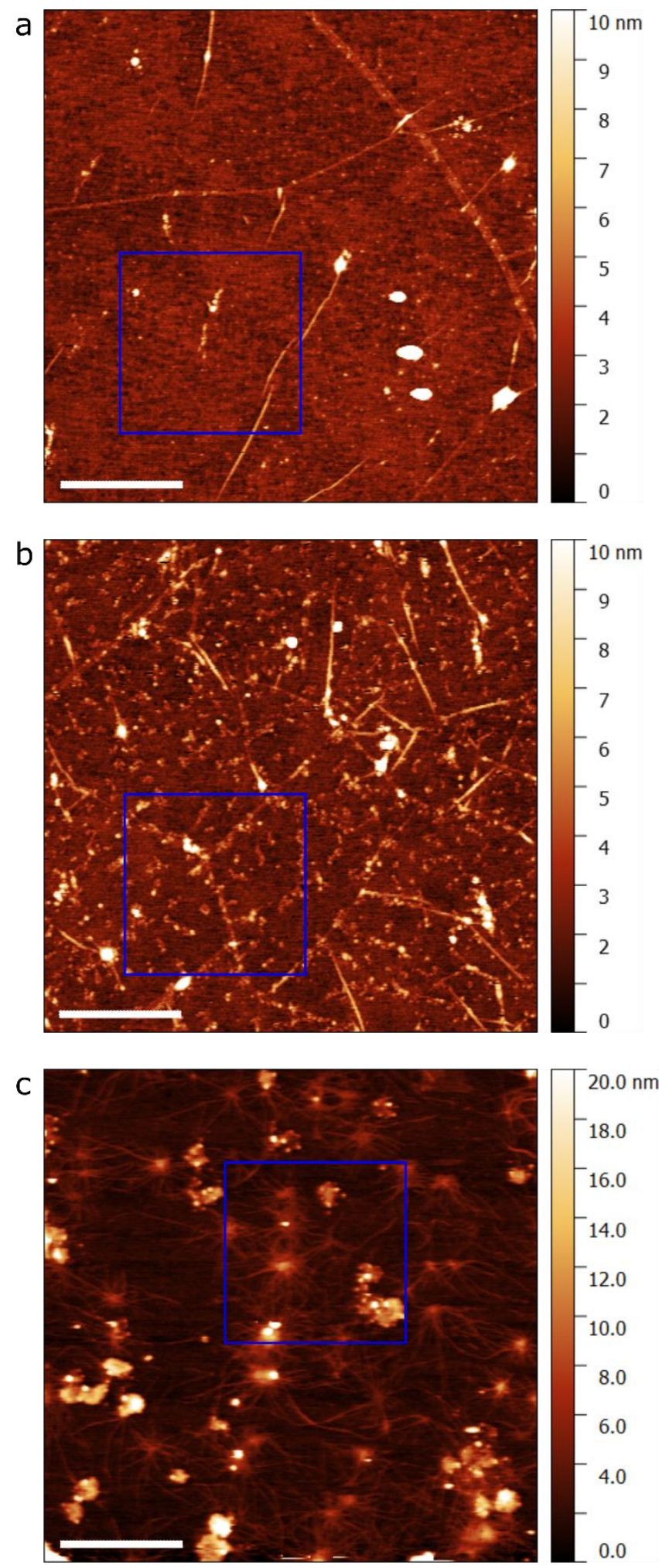

Figure 5 AFM images of (a) pristine graphene and TCNQ evaporated onto graphene/SiO2 inside the large tube evaporator with the following conditions: (b) 60 minutes at $120^{\circ}$ and (c) 120 minutes at $170^{\circ} \mathrm{C}$.

Figure 5I shows an AFM image of a sample with a very high TCNQ coverage, with 120 minutes evaporation at $170^{\circ} \mathrm{C}$. From this image it is evident that the surface morphology has changed 
significantly. Firstly, there are rather large clusters which have agglomerated, with heights ranging between 10 to $20 \mathrm{~nm}$ and typical size of several hundred $\mathrm{nm}$. The RMS of the blue square has increased to $2.0 \mathrm{~nm}$. In addition, some sort of filament or string-like feature can be seen to extend from cluster to cluster, with a typical height of 1.5 to $2.5 \mathrm{~nm}$. It is possible that these filaments are folds or wrinkles on the graphene surface caused by the TCNQ clusters, or even the intercalation of TCNQ below the graphene surface. Indeed, intercalation of various atomic elements has been observed to occur in ultra-high vacuum conditions for several graphene/metallic substrate systems ${ }^{24,25}$. Defect sites in the graphene lattice are speculated to serve as the points for the evaporated substance to intercalate between the graphene and the substrate. A similar mechanism could be at work in this case, with small defects or even larger rips and tears in the graphene serving as points of entry for the intercalation of TCNQ molecules. If the observed wrinkles are indeed occurring in the graphene as a result of the TCNQ evaporation, it would be creating stress on the graphene lattice and possibly increasing the defect density, explaining the D peak intensity increase observed in Raman measurements.

\section{CONCLUSIONS}

TCNQ was thermally evaporated onto CVD grown graphene transferred to $\mathrm{Si} / \mathrm{SiO}_{2}$ substrates. High growth temperatures and long growth times readily resulted in the formation of TCNQ crystals on the graphene surface. For increasing temperatures, the crystals changed from having a pellet shape and being spaced widely apart on the surface, to densely packed TCNQ needles. Films were formed on the graphene for low evaporation temperatures, or when the graphene/ $\mathrm{SiO}_{2}$ substrate was heated. Raman spectroscopy confirmed that the graphene films were comprised of TCNQ and DCTC, the oxidation product of TCNQ, which requires an electron charge transfer from the substrate to the TCNQ for its formation. For higher TCNQ coverages, the films only exhibited TCNQ peaks, indicating that the signal from the bulk TCNQ film overwhelms the signal from first few oxidized layers of DCTC. For very low TCNQ coverage, AFM measurements barely detected any change in the graphene surface. As coverage increased, nanometer sized clusters began to form, eventually forming clusters with heights up to $20 \mathrm{~nm}$ and typical sizes of several hundred nanometers. Filament-like ripples were seen to form between the clusters, which we speculate may be wrinkles in the graphene lattice caused by stress from the clusters, or from TCNQ molecules intercalated beneath the graphene.

\section{ACKNOWLEDGEMENTS}

Financial support by the Ministerio de Economía y Competitividad (MINECO) through grants FIS2013-40667-P, FIS2015-67367-C2-1-P and SUPERMNAN (ESP2015-65597-C4-3-R) and Comunidad de Madrid through the program MAD2D P2013/MIT-3007 is gratefully acknowledged. D.G. acknowledges RYC-2012-09864, A.B. acknowledges FPI-UAM program.

\section{REFERENCES}

1. V. Georgakilas, M. Otyepka, A. B. Bourlinos, V. Chandra, N. Kim, K. C. Kemp, P. Hobza, R. Zboril, K. S. Kim. Chemical Reviews 112, 6156-6214 (2012).

2. J. J. Navarro, S. Leret, F. Calleja, D. Stradi, A. Black, R. Bernardo-Gavito, M. Garnica, D. Granados, A. L. Vázquez de Parga, E. M. Pérez and R. Miranda. Nano Letters 16, 355-361 (2016). 
3. M. Garnica, D. Stradi, S. Barja, F. Calleja, C. Díaz, M. Alcamí, N. Martín, A. L. Vázquez de Parga, F. Martín and R. Miranda. Nature Physics 9, 368-374 (2013).

4. W. Zhang, C.-T. Lin, K.-K. Liu, T. Tite, C.-Y. Su, C.-H. Chang, Y.-H. Lee, C.-W. Chu, K.-H. Wei, J.-L. Kuo and L.-J. Li. ACS Nano 5, 7517-7524 (2011).

5. C.-L. Hsu, C.-T. Lin, J.-H. Huang, C.-W. Chu, K.-H. Wei and L.-J. Li. ACS Nano 6, 5031-5039 (2012).

6. M. Garnica, D. Stradi, S. Barja, C. Díaz, M. Alcamí, A. Arnau, A. L. Vázquez de Parga, F. Martín and R. Miranda. Nano Letters 14, 4560-4567 (2014).

7. D. Maccariello, M. Garnica, M. A. Niño, C. Navío, P. Perna, S. Barja, A. L. Vázquez de Parga, and R. Miranda. Chemistry of Materials 26, 2883-2890 (2014).

8. M. S. Khatale, J. P. Devlin. The Journal of Chemical Physics 70, 1851-1859 (1979).

9. M. Harris, J. J. Hoaglang, U. Mazur and K. W. Hipps. Vibrational Spectroscopy 9, 273-277 (1995).

10. A. Pawlukojć, I. Naktaniec, G. Bator, L. Sobczyk and E. Grech. Chemical Physics Letters, 378, 665-672 (2003).

11. X. Jiang, Z. Li, C. Sun and X. Zhang. Journal of the Physical Sciences 7, 901-905 (2012).

12. D. L. Jeanmarie and R. P. Van Duyne. Journal of the American Chemical Society 98, 4029-4033 (1976).

13. H.-H. Tseng, M. Serri, N. M. Harrison and S. Heutz. Journal of Materials Chemistry C 3, 8694 (2015).

14. M. Yoshikawa, S. Nakashima and A. Mitsuishi. Journal of Raman Spectroscopy 17, 369-371 (1986).

15. W. Chen, S. Chen, D. C. Qi, X. Y. Gao and A. T. S. Wee. Journal American Chemical Society 129, 10418-10422 (2007).

16. Y. Qi, U. Mazur and K. W. Hipps. RSC Advances 2, 10579-10584 (2012).

17. H. Alves, A. S. Molinari, H. Xie and A. Mopurgo. Nature Materials 7, 574-580 (2008).

18. H. Alves, R. M. Pinto and E. S. Maçôas. Nature Communications 4, 1842 (2013).

19. A. C. Ferrari. Solid State Communications 143, 47-57 (2007).

20. D. J. Mascaro, M. E. Thompson, H. I. Smith and V. Bulović. Organic Electronics 6, 211-220 (2005).

21. F. Yang, M. Shtein, and S. R. Forrest. Journal of Applied Physics 98, 014906 (2005). 
22. A. Das, S. Pisana, B. Chakraborty, S. Piscanec, S.K. Saha, U.V. Waghmare, K.S. Novoselov, H.R. Krishnamurthy, A.K. Geim, A.C. Ferrari and A.K. Sood. Nature Nanotechnology 3, 210-215 (2008).

23. K. Sasaki, K. Kato, Y. Tokura, S. Suzuki, T. Sogawa. Physical Review B 86, 201403 (2012).

24. S. Vlaic, A. Kimouche, J. Coraux, B. Santos, A. Locatelli and N. Rougemaille. Applied Physics Letters 104, 101602 (2014).

25. F. Calleja, H. Ochoa, M. Garnica, S. Barja, J. J. Navarro, A. Black, M. M. Otrokov, E.V. Chulkov, A. Arnau, A. L. Vázquez de Parga, F. Guinea and R. Miranda. Nature Physics 11, 43-47 (2015). 